\title{
Uma proposta para determinação da janela de média em escoamentos turbulentos a partir da decomposição de multiresolução
}

\author{
A proposition to determine window average for turbulent flows by \\ multiresolution decomposition
}

\author{
Franco Cassol ${ }^{1}$, Franciano Scremin Puhales², Otávio Costa Acevedo², Gervásio Annes \\ Degrazia $^{2}$, Vagner Anabor ${ }^{1}$, Everson Dal Piva ${ }^{1}$, Felipe Denardin Costa ${ }^{2}$ \\ ${ }^{1}$ Grupo de modelagem atmosférica de Santa Maria, RS, Brasil \\ fccassol@gmail.com, vanabor@ufsm.br; fdenardin@unipampa.edu.br \\ ${ }^{2}$ Universidade Federal de Santa Maria, RS, Brasil \\ fpuhales@gmail.com; otavio@ufsm.br; gervasiodegrazia@gmail.com; everson.bento@gmail.com
}

\begin{abstract}
Resumo
As estimativas de variáveis turbulentas utilizando o procedimento de Reynolds dependem de um intervalo de tempo definido em que as médias e as flutuações são calculadas. Esse intervalo de tempo, chamado de janela de média, é capaz de estimar as variáveis de interesse levando em conta as contribuições de movimentos com frequência igual ou superior ao inverso do próprio intervalo, portanto a janela de média deve ser adequadamente escolhida de forma que seja um intervalo suficientemente grande para representar a turbulência em todas as suas escalas, e suficientemente pequena para não levar em consideração as contribuições feitas por processos atmosféricos de baixa frequência. Neste trabalho, são apresentados os resultados da aplicação do método de decomposição em multiresolução para determinar a lacuna espectral, que é a escala de tempo que separa a turbulência dos demais processos, encontrando assim janelas de média adaptativas para a estimativa de variáveis turbulentas. Os resultados desta metodologia são comparados aos encontrados para séries de janelas fixas de 30min, 15min, $5 \mathrm{~min}$ e $1 \mathrm{~min}$. A comparação é realizada através da correlação entre a energia cinética turbulenta e a velocidade de fricção. Os resultados indicam uma melhor correlação para as variáveis quando se aplica o procedimento de janelamento adaptadivo.
\end{abstract}

Palavras-chave: Camada limite estável, Média de Reynolds, gap espectral, espectro de multiresolução.

\begin{abstract}
The estimative of turbulent quantities by Reynolds average depend on time scale average. This average window should be great enough to describe all turbulent contributions and small enough to filter the largest scales (low frequencies contributions). In this work are applied the multiresolution decomposition to find the spectral gap that split the turbulent of non-turbulent motions, this way the best time scale for the Reynolds average. The results of that methodology are compared with 1, 5, 15, and 30min windows average. This task is accomplished by the linear correlation between tubulent kinetic energy and friction velocity scale. The results reveal a better linear relationship between kinetic energy and friction velocity scale with adaptative time scale average than ones found with frozen window average.
\end{abstract}

Keywords: Stable boundary layer, Reynolds average, spectral gap, multiresolution spectrum. 


\section{Introdução}

Quando um fluido escoa próximo a uma superfície dá-se a formação de uma camada limite (Wyngaard, 2010). Por ser o ar atmosférico um fluido que escoa nas adjacências da superfície terrestre, próximo a ela ocorre a formação de uma camada limite, caracterizada pelo cisalhamento do campo de velocidade e pela influência dos processos de aquecimento e de resfriamento da superfície. A esta particular região da troposfera dá-se o nome de camada limite planetária (CLP) (Stull, 1988). Em função de suas características, o regime de escoamento na CLP é turbulento. Desta forma, pode se definir a CLP como a região da atmosfera na qual o escoamento turbulento é diretamente influenciado pela presença da superfície (Businger, 1981).

O regime de escoamento turbulento possui grande importância na atmosfera, uma vez que garante efetivos mecanismos de transporte horizontal e mistura vertical de quantidades escalares e vetoriais (Wyngaard, 2010). A turbulência consiste em um movimento irregular e quase-aleatório do fluido definida em função das rápidas flutuações na magnitude das variáveis. Devido a insolubilidade analítica da equação que descreve um escoamento turbulento, a descrição estatística do problema é largamente aplicada para melhor compreendê-lo. Neste sentido, o procedimento chamado média de Reynolds, em que a variável do escoamento turbulento é separada em uma parte média e uma parte que representa a flutuação em torno da média, é usado com o intuito de separar o comportamento médio do comportamento perturbado (turbulento) das variáveis.

Quando aplica-se o procedimento de Reynolds para separar as variáveis em uma parte média e uma parte turbulenta, pode-se definir a Energia Cinética Turbulenta $(E C T)$ em termos da variância do campo de velocidade do vento. Do ponto de vista observacional euleriano, para se calcular essa energia, ou qualquer outra variável turbulenta de interesse, é necessário escolher um intervalo de tempo para a determinação dos valores médios das variáveis, ou seja uma janela de média. Uma janela de média é capaz de estimar a variável de interesse levando em conta a contribuição de movimentos de frequência superior ao inverso do próprio intervalo de tempo escolhido. Assim, para estimar turbulência, é necessário definir as médias e as flutuações com uma janela de tempo suficientemente grande para usar a contribuição turbulenta em todas as suas escalas de tempo, e suficientemente pequena para que não considere contribuições feitas por processos de outras escalas, ou seja, processos de baixa frequência (Wyngaard, 2010; Acevedo et al., 2014).

A CLP apresenta um ciclo diário bem definido, sobretudo sobre o continente e na ausência de nuvens.
Pela manhã ocorre o aquecimento da superfície e após isso a transferência de calor para as partes mais baixas da troposfera. Tal transferência induz uma diferença de densidade que caracteriza uma condição instável. Essa camada instável, chamada de Camada Limite Convectiva, possui turbulência mais intensa e evolui para espessuras típicas de mais de $1 \mathrm{~km}$. No fim da tarde com a diminuição da radiação solar o forçante térmico se inverte, a região mais baixa passa a ser mais fria do que as mais altas o que caracteriza uma condição de equilíbrio estável, a Camada Limite Convectiva deixa de existir e forma-se a Camada Limite Estável (noturna), nessa camada o único forçante responsável por gerar turbulência é o cisalhamento do vento médio, o forçante mecânico, e sua espessura é menor do que a de uma camada convectiva (Stull, 1988).

Para estudos da camada estável, a investigação da turbulência torna-se mais complicada devido ao único forçante responsável por gerá-la ser o mecânico. Desta forma, surgem problemas tais como a turbulência não ser bem desenvolvida, a espessura da camada não ser claramente identificável, etc. Durante o dia, a turbulência faz grande parte dos transportes na CLP devido à sua maior intensidade. Já no período noturno, isto não ocorre e, por isso, as estimativas nesse período são mais influenciadas pela janela de média escolhida. Assim, boas estimativas das variáveis médias e turbulentas passam por uma escolha correta desta janela, sobretudo na camada estável.

A janela de média ideal é aquela que utiliza a lacuna espectral, que é a escala de tempo que separa a turbulência das outras escalas, para definir as médias e as flutuações. No entanto, em diferentes instantes, turbilhões de diferentes escalas dominam os movimentos. Portanto, a lacuna espectral é variável, e isso significa que qualquer intervalo de tempo fixo escolhido de alguma forma apresentaria falhas nas estimativas. A ideia de usar a lacuna espectral variável como intervalo de tempo para janela de média significa que esta janela deve ser adaptativa, variando conforme as condições dominantes em um intervalo de tempo.

O presente trabalho utiliza dados de medições em alta frequência $(60 \mathrm{~Hz})$ na Camada Limite Estável das três componentes da velocidade do vento: $u_{i}=(u, v, w)$ obtidas em sete níveis de medidas durante o experimento FLOSS (Fluxes over Snow Surfaces). Uma descrição do arranjo experimental do FLOSS pode ser encontrada no trabalho de Vickers e Mahrt (2004).

O objetivo do trabalho é propor um método de identificar a lacuna (ou gap) espectral que separa a turbulência de processos de outras escalas. Para isso foi utilizado uma ferramenta de decomposição de sinais, a decomposição em multiresolução (Vickers e Mahrt, 2003). Para verificar o impacto deste método foi avaliado o comportamento da ECT em termos da velocidade de fricção 
$\left(u_{*}\right)$, sendo estas quantidades definidas por:

$$
\begin{aligned}
u_{*} & =\left(C_{u^{\prime} w^{\prime}}^{2}+C_{v^{\prime} w^{\prime}}^{2}\right)^{1 / 4} \\
E C T & =\frac{1}{2}\left(\sigma_{u}^{2}+\sigma_{v}^{2}+\sigma_{w}^{2}\right)
\end{aligned}
$$

onde $C_{\alpha^{\prime} w^{\prime}}$ é a covariância entre as flutuações das componentes horizontal do vento e a vertical e $\sigma_{i}^{2}$ representa a variância das componentes da velocidade do vento calculadas através das janelas adaptativas e janelas fixas, arbitrariamente definidas, de $1 \mathrm{~min}, 5 \mathrm{~min}, 15 \mathrm{~min}$ e 30 min.

A velocidade de fricção é uma medida direta do gradiente vertical do campo do vento, e é estimada utilizando os fluxos cinemáticos de momentum, dados pelas covariâncias definidas na equação 1. A Energia Cinética Turbulenta é uma medida da intensidade da turbulência, já que é diretamente obtida pela variância do campo de velocidade (equação 2). Espera-se que, em um escoamento turbulento, essas duas variáveis estejam bem correlacionadas. Nesse caso, ECT e $u_{*}^{2}$ devem estar correlacionadas linearmente.

\section{Metodologia}

A decomposição em multiresolução foi implementada em séries de $2^{18}$ pontos para calcular os espectros de energia nos sub-intervalos com 2 a $2^{17}$ pontos. Neste caso considerou-se o espectro de multiresolução das componentes horizontais do vento. O resultado esperado consiste em um perfil onde o espectro da energia das componentes horizontais da velocidade vento é crescente com o aumento das escalas temporais, pois contribuições de turbilhões de maiores e mais efetivas escalas são consideradas até atingir um valor máximo local. Nesta escala o comportamento se inverte até chegar a um mínimo local, onde a energia volta a crescer. Esta região que compreende o mínimo local do espectro é o gap espectral.

A identificação dessa separação foi realizada através do seguinte algoritmo:

- determina-se o máximo local em uma escala de tempo $\tau_{i}$, comparando-se com os valores do espectro no intervalo de escalas $\left[\tau_{i-1}: \tau_{i+1}\right]$;

- determina-se o mínimo local em uma escala de tempo $\tau_{j}$, comparando-se com os valores do espectro no intervalo de escalas $\left[\tau_{j-2}: \tau_{j+2}\right.$, sendo $j \geq i]$;

- a escala de tempo $\tau_{j}$ é considerada como a escala de tempo do gap espectral.
Porém, nem sempre essa identificação é possível devido a inexistência deste comportamento. A figura 1 apresenta dois exemplos para a determinação da lacuna espectral.

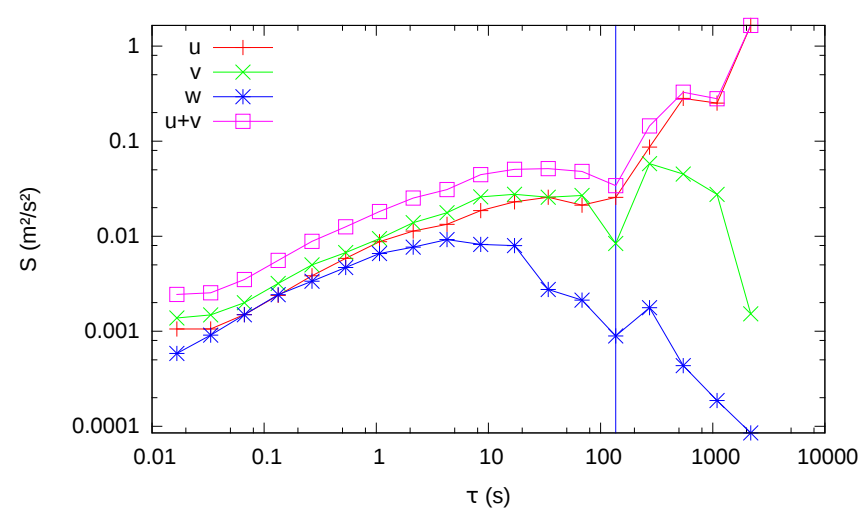

(a) gap é identificado

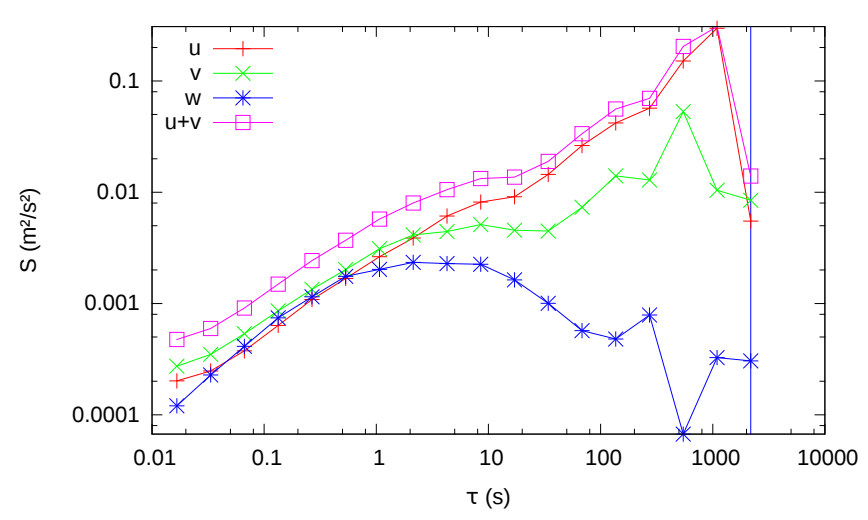

(b) gap não é identificado

Figura 1: Configurações possíveis na determinação do gap espectral de acordo com o algoritmo proposto.

Na figura 1(a), o espectro apresenta o comportamento descrito anteriormente e assim, tanto o máximo quando o mínimo local podem ser identificados. Na figura 1(b), o método proposto não encontra o comportamento de máximo e mínimo local. Nesses casos, portanto, as estimativas das variáveis de interesse usando a janela adaptativa não foram realizadas.

\section{Resultados e Discussões}

Em 55\% dos casos o método do ponto de mínimo identificou o gap espectral. A distribuição de frequência dos valores encontrados, e que foram usados como janela de média, são demonstrados na figura 2. Nesta figura, a abscissa indica os valores de $m$, associados à janela de média de $2^{m}$, identificada pelo método. Neste caso, a 
janela de média é dada por:

$$
\tau_{m}=\frac{2^{m}}{f}
$$

onde $\tau_{m}$ é a janela de média temporal e $f$ a frequência de aquisição de dados.

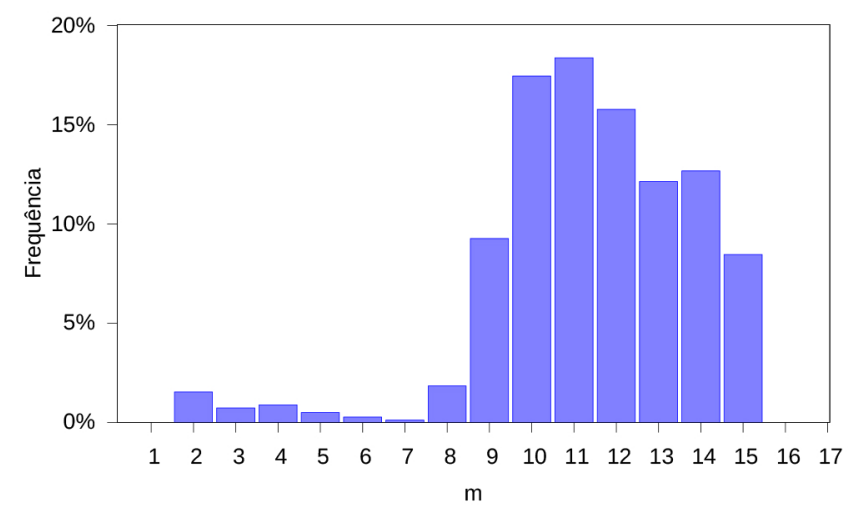

Figura 2: Distribuição de frequência do gap espectral utilizado para as estimativas.

De uma forma geral, a maior parte das janelas $(76,4 \%)$ ficaram entre $m=10$ e $m=14$ com um máximo em $m=11$. Contudo, este máximo é pouco maior que os intervalos vizinhos. Considerando o intervalo $10 \leq$ $m \leq 12$ (que corresponde a $51,6 \%$ dos casos avaliados) a janela de média variou entre 17,07 e 68,27 segundos. Vale destacar a frequência significativamente alta $(24,8 \%)$ para $m=13$ e $m=14$, equivalente janelas de 2,28min e $4,55 \mathrm{~min}$ respectivamente.

Uma reta de regressão linear deve reproduzir adequadamente a correlação entre as duas variáveis (ECT e $u_{*}^{2}$ ). As figuras 3 e 4 mostram o comportamento da energia cinética turbulenta em função da velocidade de fricção. Além disso, uma reta do tipo $g(x)=a x$ é ajustada a cada conjunto de dados a fim de avaliar sua correlação linear. Nas figuras 3(a) e 3(b) é possível observar um maior espalhamento dos dados e uma fuga ao comportamento de lei de potência entre as duas variáveis, para janelas fixas de 15 e $30 \mathrm{~min}$. No entanto, para as janelas fixas de períodos menores ( $5 \mathrm{~min}$ - figura 3(c), e especialmente $1 \mathrm{~min}$ - figura 3(d)) e também para a janela adaptativa (figura 4) um comportamento mais característico de uma função linear é observado.

A tabela 1 apresenta uma sumarização dos coeficientes angulares das retas em cada um dos gráficos das figuras 3 e 4 , bem como o coeficiente de correlação linear do ajuste.

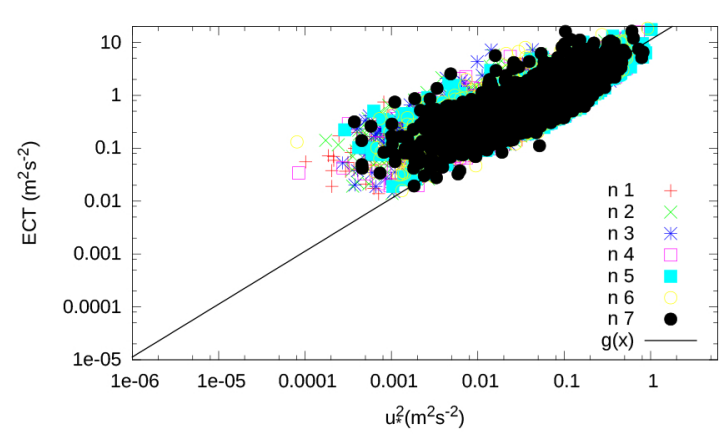

(a) Janela de 30 minutos

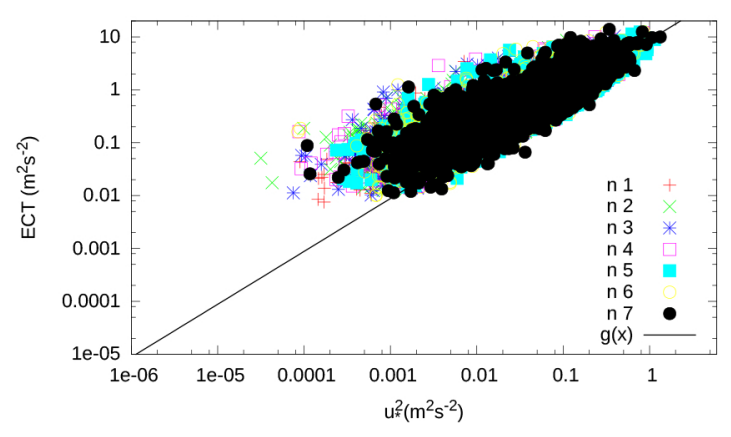

(b) Janela de 15 minutos

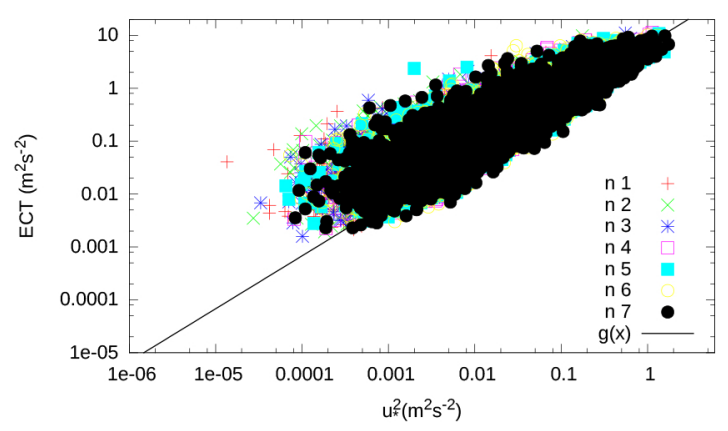

(c) Janela de 5 minutos

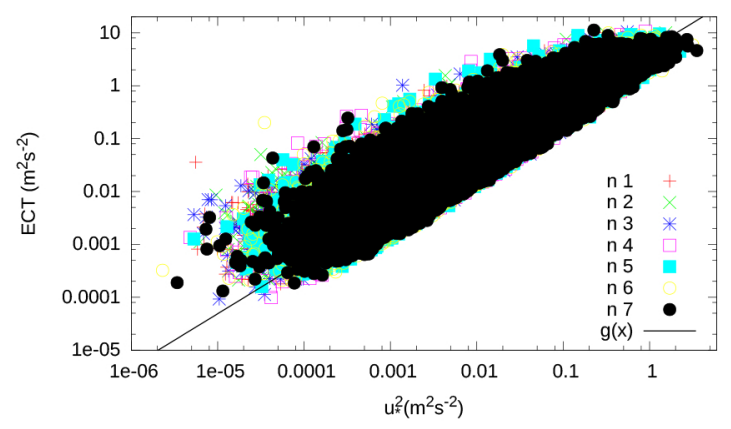

(d) Janela de 1 minutos

Figura 3: Avaliação do comportamento $E C T \times u_{*}^{2}$ para as janelas fixas. 


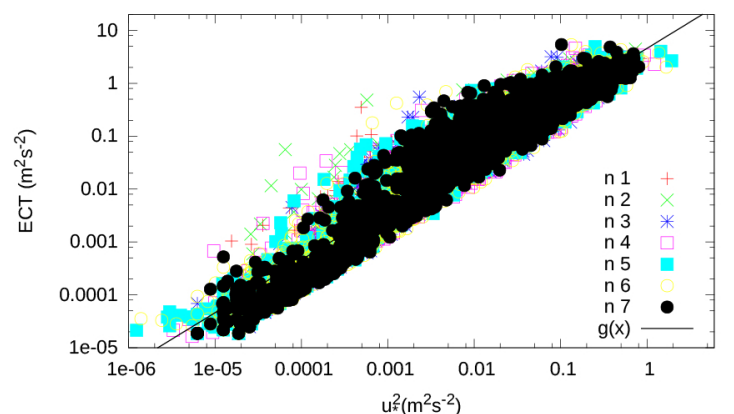

Figura 4: Avaliação do comportamento $E C T \times u_{*}^{2}$ para as janelas adaptativas.

Tabela 1: Resultados do ajuste linear dos dados obtidos.

\begin{tabular}{ccc}
\hline janela & $a$ & $R$ \\
\hline $30 \mathrm{~min}$ & 11,25 & 0,69 \\
$15 \mathrm{~min}$ & 8,78 & 0,75 \\
$5 \mathrm{~min}$ & 6,78 & 0,83 \\
$1 \mathrm{~min}$ & 4,88 & 0,87 \\
adaptativa & 4,58 & 0,87 \\
\hline
\end{tabular}

Essas diferenças entre as correlações para as diferentes janelas de médias, demostram que as estimativas pelas janelas fixas arbitrárias de maiores períodos são muito influenciadas por processos de baixa frequência, não turbulentos, também denominados submeso (Acevedo et al., 2014), enquanto que as fixas de menor período atingem as escalas temporais mais usuais da turbulência em camada estável. De fato, isso é comprovado na figura 2, pois os valores típicos filtrados para o gap espectral estão, em geral, na faixa das janelas fixas arbitrariamente escolhidas de menor período.

\section{Considerações finais}

Os resultados apresentados mostram uma boa eficácia do método proposto para adequar a janela de média para estimativas em turbulência, quando avalia-se a correlação entre a energia cinética turbulenta e a velocidade de fricção. Contudo, ainda é necessário avaliar se existe alguma dependência com a altura e intensidade da turbulência (regimes muito estáveis e fracamente estáveis). Além disso, é preciso avaliar a correlação entre outras variáveis, tais como fluxos turbulentos de grandezas escalares e vetoriais.

Quando comparados com os resultados obtidos pelas médias de janela fixa, os resultados da janela adaptativa se aproximam dos resultados para as menores janelas. Isto porque, mesmo que seja variável, a janela adaptativa tem seu valor médio da ordem de um minuto. Além disso, Acevedo et al. (2014) mostraram com dados do experimento FLOSS que, na média, os processos de submeso tem escala de tempo a partir de 10 s até aproximadamente 100s, dependendo da intensidade da turbulência. Cabe aqui salientar que os resultados deste trabalho concordam com os resultados de Acevedo et al. (2014), uma vez que a maior parte das janelas de média determinadas também estão dentro deste intervalo.

O método se baseia em condições específicas, e de certa forma idealizadas, do perfil do espectro de energia cinética turbulenta. Por isso, em vários casos em que o perfil do espectro não segue a tendência ideal, o método não separa a escala turbulenta das outras escalas, e as estimativas não são realizadas. Para completar a separação entre turbulência e outros processos, deveria ser usada uma outra forma de encontrar uma escala de tempo equivalente a do gap espectral, alternativamente ao método proposto. Desta forma, tal investigação é proposta aqui para trabalhos futuros.

\section{Referências}

Acevedo, O. C., Costa, F. D., Oliveira, P. E., Puhales, F. S., Degrazia, G. A., Roberti, D. R. (2014). The influence of submeso processes on stable boundary layer similarity relationships. Journal of the Atmospheric Sciences, 71(1), 207-225.

Businger, J. A. (1981). Equations and concepts. Em: Nieuwstadt, F., Dop, H. V. (Eds) Atmospheric turbulence and air pollution modeling, D. Reidel Publishing Company, Dordrecht, Cap. 1, pp. 1-36.

Stull, R. (1988). An introduction to boundary layer meteorology. Kluwer Academic Publishers, Dordrecht.

Vickers, D., Mahrt, L. (2003). The cospectral gap and turbulent flux calculations. Journal of Atmospheric and Oceanic technology, 20(5), 660-672.

Vickers, D., Mahrt, L. (2004). Evaluating formulations of stable boundary layer height. Journal of applied meteoro$\log y, 43(11), 1736-1749$.

Wyngaard, J. C. (2010). Turbulence in the atmosphere. Cambridge University Press, Cambridge. 